
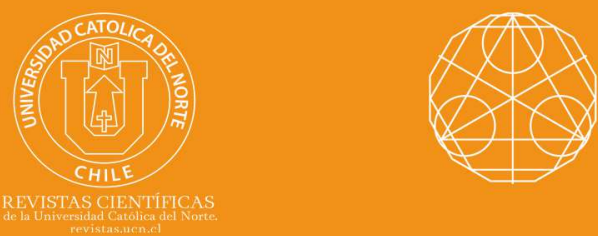

\title{
On independent position sets in graphs
}

Elias John Thomas ${ }^{1}$ (1) orcid.org/0000-0003-0598-4726

Ullas Chandran S. V. ${ }^{2}$ [D orcid.org/0000-0002-2081-9094

${ }^{1}$ Mar Ivanios College, University of Kerala, Dept. of Mathematics, Thiruvananthapuram, KL, India.

eliasjohnkalarickal@gmail.com

${ }^{2}$ Mahatma Gandhi College, University of Kerala, Dept. of Mathematics, Thiruvananthapuram, KL, India.

『svuc.math@gmail.com

Received: April 2020 | Accepted: October 2020

\section{Abstract:}

An independent set $S$ of vertices in a graph $G$ is an independent position set if no three vertices of $S$ lie on a common geodesic. An independent position set of maximum size is an ip-set of $G$. The cardinality of an ip-set is the independent position number, denoted by ip(G). In this paper, we introduce and study the independent position number of a graph. Certain general properties of these concepts are discussed. Graphs of order $n$ having the independent position number 1 or $n-1$ are characterized. Bounds for the independent position number of Cartesian and Lexicographic product graphs are determined and the exact value for Corona product graphs are obtained. Finally, some realization results are proved to show that there is no general relationship between independent position sets and other related graph invariants.

Keywords: General position set; Independent set; Independent number; Independent position number.

MSC (2020): 05C12, 05C69.

Cite this article as (IEEE citation style):

E. J. Thomas and U. Chandran S. V., "On independent position sets in graphs", Proyecciones (Antofagasta, On line), vol. 40,

no. 2, pp. 385-398, 2021, doi: 10.22199/issn.0717-62792021-02-0023

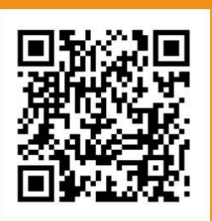

Article copyright: (C) 2021 Elias John Thomas and Ullas Chandran S. V. This is an open access article distributed under the terms of the Creative Commons License, which permits unrestricted use and distribution provided the original author and source are credited. 


\section{Introduction}

The general position problem in a graph $G$ is to find the maximum size of a set $S$ of vertices in $G$ such that no three verices of $S$ lie on a common shortest path. The general position problem in graph theory was introduced and studied in [1] after getting inspiration from the century old Dudeney's no-three-in-line problem [2] and by the general position subset selection problem $[3,4]$ from discrete geometry. But the same concept has already been studied two years earlier in [5] under the name geodetic irredundant sets. Refer $[6,7,8,9,10,11]$ to understand the recent developments on general position number.

Graphs in this paper are finite, undirected and simple. The distance between two vertices $u$ and $v$ in a connected graph $G$ is the length of a shortest $u, v$-path in $G$, denoted as $d(u, v)$. A $u, v$-geodesic is a $u, v$-path of length $d(u, v)$. Obviously the distance is a metric on the vertex set $V$. The maximum distance between all pairs of vertices of $G$ is the diameter, $\operatorname{diam}(G)$ of $G$. The interval $I_{G}[u, v]$ between vertices $u$ and $v$ of a graph $G$ is the set of vertices $x$ such that there exists a $u, v$-geodesic which contains $x$. For a positive integer $n$, let $[n]=1, \ldots, n$.

A set of vertices $S \subseteq V(G)$ is a general position set of $G$ if no three vertices of $S$ lie on a common geodesic. A gp-set is thus a largest general position set. The general position number (gp-number for short) of $G$ is the cardinality of a gp-set of $G$. An independent set or a stable set $S$, is a set of vertices in a graph such that no two vertices of $S$ are adjacent. The vertex independence number of a graph ( or simply, independence number) is the cardinality of the largest independent vertex set. The independence number is most commonly denoted by $\alpha(G)$.

In this paper, we investigate the properties of a graph invariant named independent position number which admits both the properties of general position set and independence set. In the following section definitions and preliminary observations are listed. In Section 3, we prove some bounds for independent position number on Cartesian product, lexicographic product and an exact value for corona product of graphs. In section 4, we prove some realization results which shows that the independent position number is different from other related invariants.

In this paper, we make use of the following results.

Theorem 1.1. [5] For a connected graph $G$ of order $n$ and diameter $d$, $g p(G) \leq n-d+1$. 
Theorem 1.2. [5] For a connected graph $G, g p(G)=2$ if and only if $G=P_{n}$ or $G=C_{4}$.

Theorem 1.3. [5] For any tree $T$ with $k$ end vertices, $g p(T)=k$.

\section{Independent general position sets in graphs}

A set $S$ of vertices in a graph $G$ is considered to be an independent position set if $S$ is both an independent set and a general position set in $G$. An independent position set of maximum size is called an $i p$-set of $G$. The cardinality of an $i p$-set is called the independent position number, denoted by $i p(G)$. It is clear that every independent position set is a general position set and so we have the following observation.

Observation 2.1. For any connected graph $G$ of order $n, 1 \leq i p(G) \leq$ $g p(G) \leq n$.

Example 2.2. For the graph $G$ in Figure 1, the set $S=\left\{v_{1}, v_{2}, v_{3}, v_{4}\right\}$ is a maximum general position set of $G$. Then $g p(G)=4$. On the other hand, any independent position set of $G$ contains at most one vertex from the set $\left\{v_{1}, v_{2}, v_{3}, v_{4}\right\}$ and so $i p(G) \leq 3$. Now, since the set $T=\left\{v_{5}, v_{1}, v_{6}\right\}$ is an independent position set, it follows that $i p(G)=3$.

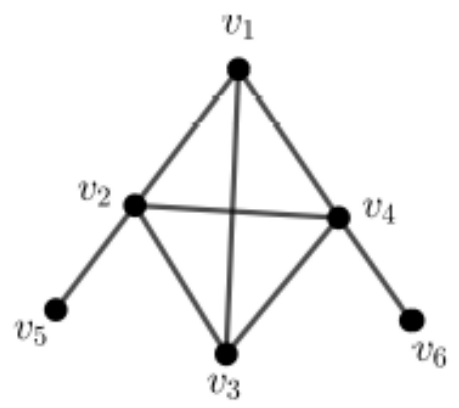

Figure 1

Proposition 2.3. For a connected graph $G$ of order $n$ and diameter $d$, $i p(G) \leq n-d+1$.

Proof.This follows from Theorem 1.1 and Observation 2.1. 
Proposition 2.4. . For any connected graph $G$ of order $n, 1 \leq i p(G) \leq$ $\alpha(G)$.

Proof. The left inequality is trivial and the right inequality follows from the fact that every independent position set is an independent set. The independent position number can be significantly small while compared to the independent number. To certain instant, consider the path $P_{n}(n \geq 5)$. Then it is clear from Theorem 1.2 and Observation 2.1 that $i p(G)=2$, where as $\alpha(G)=\left\lceil\frac{n}{2}\right\rceil$.

Theorem 2.5. Let $G$ be a connected graph with diameter atmost 3. Then $i p(G)=\alpha(G)$.

Proof. By Proposition 2.4, it remains to prove that $i p(G) \geq \alpha(G)$. For, let $S$ be a maximum independent set of $G$. Assume the contrary that there exists three vertices $x, y$ and $z$ in $S$ such that $y \in I_{G}[x, z]$. Let $P: x=x_{0}, x_{1}, \ldots, x_{i}=y, x_{i+1}, \ldots, x_{n}=z$ be an $x, z$ - geodesic in $G$ containing the vertex $y$. Now, since $S$ is an independent set, it follows that $2 \leq i \leq n-2$. This shows that $d_{G}(x, z) \geq 4$, a contradiction to the fact that $\operatorname{diam}(G) \leq 3$. Hence $S$ must be a general position set of $G$ and so $i p(G) \geq|S|=\alpha(G)$.

The converse need not be true. Consider the graph in Figure 2. Then $\operatorname{diam}(G)=4$ whereas, the set $\left\{v_{2}, v_{3}, v_{5}, v_{6}\right\}$ is a maximum independent set which is also a general position set. Hence $i p(G)=4=\alpha(G)$.

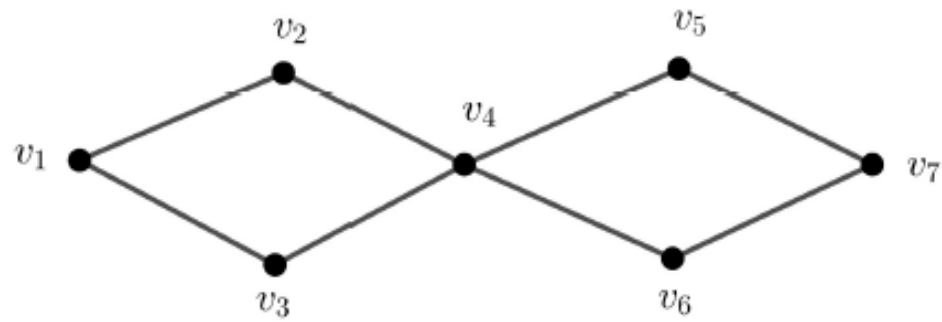

Figure 2

In view of Theorem 2.5, we have the following open problem. 
Problem 2.6. Chracterize the classes of graphs in which ip $(G)=\alpha(G)$.

Theorem 2.7. For any tree $T$ with $k$ end vertices, $i p(T)=k$.

Proof. Since the set of all end vertices of $T$ is an independent position set, it follows that $i p(T) \geq k$. Now, it follows from Theorem 1.3 and Obsevation 2.1 that $i p(T) \leq g p(T)=k$.

Theorem 2.8. Let $G$ be a connected graph of order $n$. Then

1. ip $(G)=1$ if and only if $G \cong K_{n}$.

2. $i p(G)=n-1$ if and only if $G \cong K_{1, n-1}$.

\section{Proof.}

1. If $G \cong K_{n}$, then it is obvious that $i p(G)=1$. On the other hand, let $i p(G)=1$. If $G$ is non-complete, then there exists vertices $u$ and $v$ in $G$ such that $u, v \notin E(G)$. Then $\{u, v\}$ is an independent position set and so $i p(G) \geq 2$, impossible. Hence $G \cong K_{n}$.

2. First, suppose that $i p(G)=n-1$. Then it follows from Proposition 2.3 that $\operatorname{diam}(G) \leq 2$. Since, the complete graph $K_{n}$ has independent position number 1 , it follows that $\operatorname{diam}(G)=2$. Let $S=$ $\left\{v_{1}, v_{2}, \ldots, v_{n-1}\right\}$ be an independent set of size $n-1$ and $v \in V(G)$ such that $v \notin S$. Now, since $S$ is an independent set and $G$ is connected, we have that $v$ must be adjacent to all the vertices of $S$. Then $G \cong K_{1, n-1}$. On the other hand, if $G \cong K_{1, n-1}$ then it is evident that $i p(G)=n-1$.

Theorem 2.9. If $G$ is a bipartite graph, then $i p(G)=g p(G)$.

Proof. If $G=P_{n}$ or $G=C_{4}$, then one can easily verify that $i p(G)=$ $g p(G)=2$. So in the following we may assume that $G$ is neither a path nor a $C_{4}$, so that $g p(G) \geq 3$. Now, let $S$ be a maximum general position set of $G$. Let $S_{1}, S_{2}, \ldots, S_{k}(k \geq 1)$ be the components of the induced subgraph of $S$ in $G$. Since $G$ is bipartite and each $S_{i}(1 \leq i \leq k)$ induces a clique, it follows that $\left|S_{i}\right| \leq 2$ for all $i \in[k]$. This shows that $k \geq 2$. In the following, we claim that $\left|S_{i}\right|=1$ for all $i \in[k]$. Assume the contrary that $S_{1}$ contains two vertices, say $u$ and $v$. Since $S_{1}$ induces a clique in $G$, we have that $u$ and $v$ are adjacent in $G$. Let $w$ be any vertex in $S_{2}$. Since $S$ is a general position 
set, it follows that $d(u, w)=d(v, w)$. Now, let $P: w=x_{1}, x_{2}, \ldots, x_{r}=u$ be a $w, u$-geodesic in $G$ and let $Q: w=y_{1}, y_{2}, \ldots, y_{r}=v$ be a $w, v$-geodesic in $G$. Let $t$ be the largest suffix such that $x_{t} \in V(Q)$. Since $d(u, w)=d(v, w)$, it follows that $x_{t}=y_{t}$ and hence the $x_{t}, u$-subpath of $P$ and $y_{t}, v$-subpath of $Q$ together with the edge $u v$ forms an odd cycle in $G$. This is impossible in a bipartite graph. This shows that $\left|S_{i}\right|=1$ for all $i \in[k]$. Hence $S$ is an independent general position set in $G$ and so $i p(G) \geq|S|=g p(G)$. Now, Obsevation 2.1 implies that $i p(G)=g p(G)$.

The converse of the above theorem need not be true. For, consider the non-bipartite graph shown in the Figure 3 . Then the set $S=\left\{u_{2}, u_{3}, u_{5}, u_{6}\right\}$ is an independent position set in $G$. On the other hand, any general position set of $G$ contains at most two vertices from each component of $G \backslash u_{4}$. This shows that $S$ is a maximum independent position set as well as a maximum general position set of $G$. Thus $g p(G)=i p(G)$.

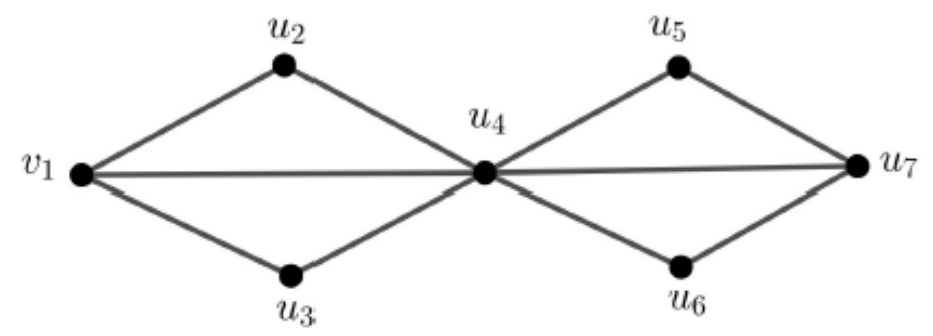

Figure 3

\section{Independent position number on some graph operations}

In the following section, we determine some bounds of independent position number on cartesian product, lexicographic product and an exact value for corona product of graphs. For more on the product graphs see [12]. 


\subsection{Cartesian products}

The Cartesian product $G \square H$ of graphs $G$ and $H$ has the vertex set $V(G) \times$ $V(H)$ and the edge set $E(G \square H)=\left\{(g, h)\left(g^{\prime}, h^{\prime}\right): g g^{\prime} \in E(G)\right.$ and $h=h^{\prime}$, or, $g=g^{\prime}$ and $\left.h h^{\prime} \in E(H)\right\}$. If $(g, h) \in V(G \square H)$, then the $G$-layer $G^{h}$ through the vertex $(g, h)$ is the subgraph of $G \square H$ induced by the vertices $\left(g^{\prime}, h\right): g^{\prime} \in V(G)$. Similarly, the $H$-layer $H^{g}$ through $(g, h)$ is the subgraph of $G \square H$ induced by the vertices $\left(g, h^{\prime}\right): h^{\prime} \in V(H)$. It is well-known that for given vertices $u=\left(g_{1}, h_{1}\right)$ and $v=\left(g_{2}, h_{2}\right)$ of $G \square H$ we have $d_{G \square H}(u, v)=$ $d_{G}\left(g_{1}, g_{2}\right)+d_{H}\left(h_{1}, h_{2}\right)$.

The announced lower bound reads as follows.

Theorem 3.1. For any connected graphs $G$ and $H, i p(G \square H) \geq i p(G)+$ $i p(H)-2$.

Proof. Let $S$ and $T$ be the maximum independent position sets of $G$ and $H$ respectively. Let $g \in S$ and $h \in T$ and $S_{1}=\{(x, h) \mid x \in S\}$ and $T_{1}=\{(g, y) \mid y \in T\}$. We claim that $W=\left(S_{1} \cup T_{1}\right) \backslash\{(g, h)\}$ is an independent position set in $G \square H$. First, we prove that $W$ is an independent set in $G \square H$. Since $S$ and $T$ are independent set in $G$ and $H$ respectively, it follows that both $S_{1}$ and $T_{1}$ are independent sets in $G \square H$. Let $(x, h) \in S_{1}$ and $(g, y) \in T_{1}$. Then it is clear that $x \neq g$ and $y \neq h$ and so by definition it follows that $(x, h)$ is not adjacent to $(g, y)$. Thus there is no adjacency between the vertices of $S_{1}$ and $T_{1}$ in $G \square H$. This shows that $W$ is an independent set in $G \square H$. Next, we claim that $W$ is a general position set in $G \square H$. Since layers are convex sets in $G \square H$, it follows that both $S_{1}$ and $T_{1}$ are general position sets in $G \square H$. Now, suppose there exist vertices $(x, h),(u, v),(g, y) \in$ $W$ such that $(u, v) \in I_{G \square H}[(x, h),(g, y)]$. Without loss of generality, we may assume that $(u, v) \in S_{1}$. Then $(u, v) \neq(g, h)$ and $v=h$. Let $P$ : $(x, h)=\left(x_{1}, y_{1}\right),\left(x_{2}, y_{2}\right), \ldots,\left(x_{i}, y_{i}\right)=(u, h),\left(x_{i+1}, y_{i+1}\right), \ldots,\left(x_{n}, y_{n}\right)=$ $(g, y)$ geodesic in $G \square H$ containing the vertex $(u, v)$. Since $G$-layer $G^{h}$ is a convex set in $G \square H$, it follows that $y_{1}=y_{2}=\ldots=y_{i}=h$. Thus $x_{j} \neq g$ for all $j=[i]$. Let $k$ be the largest suffix such that $y_{k}=h$. Thus $x_{k+1}=$ $x_{k+2} \ldots=x_{n}=g$. This shows that $x=x_{1}, x_{2}, \ldots, x_{i}=u, \ldots x_{n}=g$ is a $x, g$-geodesic in $G$ containing the vertex $u$ with $x, u, g \in S$. This is a contradiction to the fact that $S$ is a general position set, Thus $W$ must be a general position set in $G \square H$ and $i p(G \square H) \geq|W|=i p(G)+i p(H)-2$. 


\subsection{Lexicographic products}

The lexicographic product $G[H]$ of two graphs $G$ and $H$ has vertex set $V(G) \times V(H)$ and two vertices $\left(u_{1}, v_{1}\right)$ and $\left(u_{2}, v_{2}\right)$ are adjacent whenever $u_{1} u_{2} \in E(G)$, or $u_{1}=u_{2}$ and $v_{1} v_{2} \in E(H)$. To prove the bound on Lexicographic products, we may make use of the following Lemma.

Lemma 3.2. [13] Let $G$ and $H$ be graphs. Then

1. The graph $G[H]$ is connected if and only if $G$ is connected.

2. The lexicographic product is associative but not commutative.

3. If $G$ is connected, then $d_{G[H]}\left((g, h),\left(g^{\prime}, h^{\prime}\right)\right)=d_{G}\left(g, g^{\prime}\right)$ if $g \neq g^{\prime}$ $d_{G[H]}\left((g, h),\left(g, h^{\prime}\right)\right)=2$ if $h h^{\prime} \notin E(H)$ $d_{G[H]}\left((g, h),\left(g, h^{\prime}\right)\right)=1$ if $h h^{\prime} \in E(H)$

Theorem 3.3. For any connected graphs $G$ and $H, i p(G[H]) \geq i p(G) i p(H)$.

Proof. Let $S$ be an $i p$-set of $G$ and $T$ be an $i p$-set of $H$. We first show that $S \times T$ is an independent position set in $G[H]$. For, suppose that the vertices $\left(g_{1}, h_{1}\right)$ and $\left(g_{2}, h_{2}\right)$ in $S \times T$ are adjacent in $G[H]$, then we have either $g_{1} g_{2} \in E(G)$ or $h_{1} h_{2} \in E(G)$. This is impossible. Thus $S \times T$ is an independent set in $G[H]$. Next, we claim that $S \times T$ is a general position set in $G[H]$. Assume on contrary that there exist $\left(g_{1}, h_{1}\right),\left(g_{2}, h_{2}\right),\left(g_{3}, h_{3}\right) \in$ $S \times T$ such that $\left(g_{2}, h_{2}\right) \subseteq I_{G[H]}\left[\left(g_{1}, h_{1}\right),\left(g_{3}, h_{3}\right)\right]$. First, suppose that $\left(g_{1}, h_{1}\right),\left(g_{3}, h_{3}\right)$ belongs to the same $H$-layer $H^{g}$. Thus $g_{1}=g_{3}=g$ and $d_{G[H]}\left[\left(g, h_{1}\right),\left(g, h_{3}\right)\right]=2$. In this case $\left(g_{2}, h_{2}\right)$ is adjacent with both $\left(g_{1}, h_{1}\right)$ and $\left(g_{3}, h_{3}\right)$, a contradiction to the fact that $S \times T$ is an independent set. Hence $g_{1} \neq g_{3}$. Let $\left(g_{1}, h_{1}\right)=\left(x_{1}, y_{1}\right),\left(x_{2}, y_{2}\right), \ldots,\left(x_{i}, y_{i}\right)=$ $\left(g_{2}, h_{2}\right), \ldots,\left(x_{n}, y_{n}\right)=\left(g_{3}, h_{3}\right)$ be a $\left(g_{1}, h_{1}\right),\left(g_{3}, h_{3}\right)$ - geodesic contains the vertex $\left(g_{2}, h_{2}\right)$. Then by Lemma $3.2 d_{G}\left(g_{1}, g_{3}\right)=d_{G[H]}\left[\left(g_{1}, h_{1}\right),\left(g_{3}, h_{3}\right)\right]$ and so $g_{1}=x_{1}, x_{2}, \ldots, x_{i}=g_{2}, \ldots, x_{n}=g_{3}$ is a $g_{1}, g_{3}$-geodesic in $G$ containing the vertex $g_{2}$. This is a contradiction. Hence $S \times T$ is an independent position set in $G[H]$. So $i p(G[H]) \geq|S \times T|=i p(G) i p(H)$.

The bound is sharp. Consider the graph $C_{4}\left[K_{2}\right]$ shown in the Figure 4. 


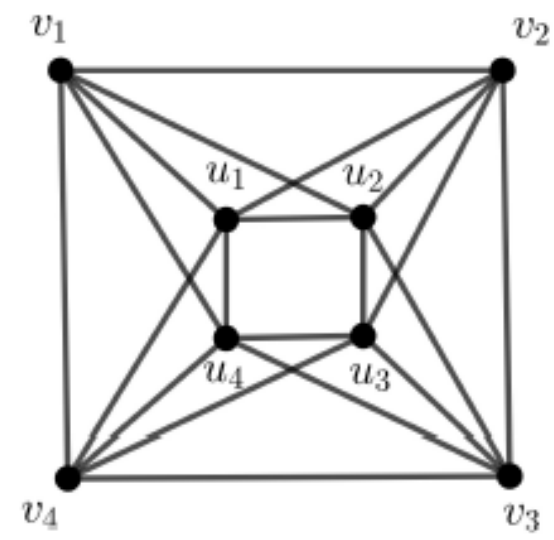

Figure 4

Clearly from the Figure 4, we can choose atmost two vertices $v_{1}, v_{3}$ or $v_{2}, v_{4}$ or $u_{1}, u_{3}$ or $u_{2}, u_{4}$ from the every layer. If we choose only one vertex from first layer say, $v_{1}$ then the possible choice of vertex from the second layer is $u_{3}$ only, since $v_{1}$ is adjacent to $v_{2}, v_{4}, u_{1}, u_{2}, u_{4}$. Therefore, $i p\left(C_{4}\left[K_{2}\right]\right)=|S \times T|=2=|S| \times|T|=i p\left(C_{4}\right) i p\left(K_{2}\right)$.

\subsection{Corona of graphs}

Let $G$ and $H$ be graphs where $V(G)=\left\{v_{1}, \ldots, v_{n(G)}\right\}$. The corona $G \circ H$ of graphs $G$ and $H$ is obtained from the disjoint union of $G$ and $n(G)$ disjoint copies of $H$, say $H_{1}, \ldots, H_{n(G)}$, where for all $i \in[n(G)]$, the vertex $v_{i} \in V(G)$ is adjacent to each vertex of $H_{i}$.

Theorem 3.4. For any graphs $G$ and $H, i p(G \circ H)=n(G) \alpha(H)$.

Proof. Let $V(G)=\left\{v_{1}, v_{2}, \ldots, v_{n}\right\}$ and let $H_{1}, H_{2}, \ldots, H_{n}$ be the corresponding copies of $H$ in $G \circ H$. For each $i=[n]$, let $S_{i}$ denote the maximum independent set in $H$. Then the set $S=S_{1} \cup S_{2} \ldots \cup S_{n}$ is an independent position set in $G \circ H$. Hence $i p(G \circ H) \geq|S|=n(G) \alpha(H)$. On the other hand, let $T$ be the maximum general position set in $G \circ H$. Then $|T| \geq n(G) \alpha(H)$. 
Claim: $T \subseteq \bigcup_{i=1}^{n} H_{i}$.

Suppose that there exists a vertex $v_{i} \in T$ for some $i$ with $1 \leq i \leq n$. Then since $T$ is an independent set, we have that $V\left(H_{i}\right) \cap T=\emptyset$. Moreover, the set $\left(T \backslash\left\{v_{i}\right\}\right) \cup S_{i}$ is an independent position set of size atleast the size of $T$. Hence without loss of generality, we may assume that $T \subseteq \bigcup_{i=1}^{n} H_{i}$. Moreover, since $T$ is an independent set, we have that $T \cap H_{i}$ is also an independent subset of $H_{i}$. Then $\left|T \cap H_{i}\right| \leq \alpha(H)$. Hence $|T|=\sum_{i=1}^{n}\left|H_{i}\right| \leq n(G) \alpha(H)$. This shows that $i p(G \circ H)=n(G) \alpha(H)$.

\section{Some Realization Results}

Here, we prove some realization results which shows that the independent position number is different from other related invariants.

Theorem 4.1. For integers $k$ and $n$ with $1 \leq k<n$, there is a connected graph $G$ of order $n$ such that $i p(G)=k$.

Proof. If $k=1$ or $k=n-1$, obviously the graphs $K_{n}$ and $K_{1, n-1}$ respectively having the desired properties. If $k=n-2$, then it follows from Theorem 2.7 that $i p(T)=n-2$ for any tree $T$ with diameter 3 . So, assume that $2 \leq k \leq n-3$. Let $G$ be the graph obtained from the cycle $C_{(n-k+2)}: v_{1}, v_{2}, \ldots, v_{(n-k+2)}, v_{1}$ by adding $k-2$ new vertices $u_{1}, u_{2}, \ldots, u_{(k-2)}$ and joining each $u_{i}$ to both $v_{1}$ and $v_{(n-k+2)}$. The graph $G$ is shown in the Figure 5.

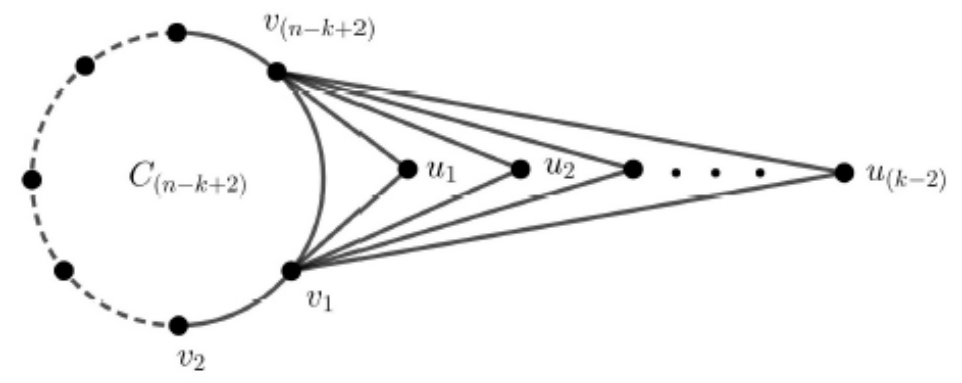

Figure 5 
From the graph $G$ in Figure 5, it is clear that $|G|=n-k+2+k-2=n$. We claim that $i p(G)=k$, it is clear that any independent position set contains at most two vertices from the cycle. Thus ip $(G) \leq n-(n-k+$ $2)+2=k$. Now, on the other hand, the set $M=\left\{u_{1}, u_{2}, \ldots, u_{(k-2)}, v_{i}, v_{m}\right\}$ with $i, m \in\{1, n-k+2\}$ is an $i p$-set in $G$, we have that $i p(G)=k$.

Theorem 4.2. For every pair of positive integers $a, b$ with $2 \leq a \leq b<n$, there exists a connected graph $G$ with $i p(G)=a$ and $g p(G)=b$.

Proof. We consider the following two cases.

Case 1. $a=b$. In this case, the graph $G$ of order $n$ with desired properties is constructed as follows. Let $G$ be the graph obtained from the path $P: v_{1}, v_{2}, \ldots, v_{(n-a+2)}$ by adding $(a-2)$ new vertices $\left\{u_{1}, u_{2}, \ldots, u_{(a-2)}\right\}$ and joining each $u_{i}$ to the vertex $v_{3}$. The graph $G$ is shown in the Figure 6 . Then, from Theorem 2.7 and Theorem 1.3 we get that $i p(G)=g p(G)=a$.

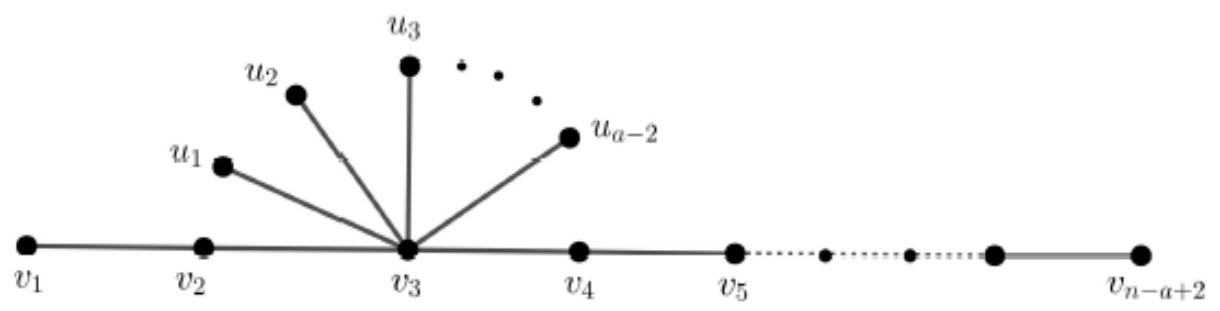

Figure 6

Case 2. $2<a<b<n$

Let $H$ be the graph obtained from the complete graph $K_{(b-a+2)}$ : $w_{1}, w_{2}, \ldots, w_{(b-a+2)}$ and a vertex disjoint path $P: u_{0}, u_{1}, u_{2}, \ldots, u_{(n-b-1)}$ by identifying the vertices $w_{1}$ and $u_{0}$. Let $G$ the graph obtained from the graph $H$ by adding $a-1$ new vertices $v_{1}, v_{2}, \ldots, v_{(a-1)}$ and join each $v_{i}$ to the vertex $u_{(n-b-1)}$. The graph $G$ of order $n$ is shown in the Figure 7 . 


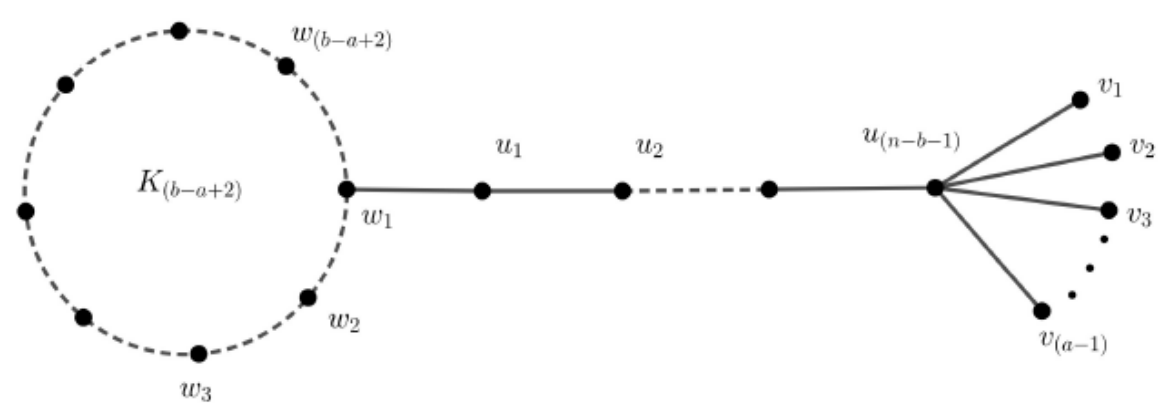

Figure 7

Let $S$ be a maximum general position set of $G$. Then $S$ contains at most two vertices from the set $\left\{w_{1}, u_{1}, u_{2}, \ldots, u_{(n-b-1)}, v_{1}\right\}$. This shows that $|S| \leq b$. Now, on the other hand, since the set

$\left\{w_{2}, w_{3}, \ldots, w_{(b-a+2)}, v_{1}, v_{2}, \ldots, v_{(a-1)}\right\}$ is a maximum general position set in $G$, it follows that $g p(G)=|S|=a-1+(b-a+1)=b$.

Next, we prove that $i p(G)=a$. Let $T$ be any independent position set. Then $T$ contains at most one vertex from the the set $\left\{w_{1}, w_{2}, \ldots, w_{(b-a+2)}\right\}$. Also, $T$ contains at most two vertices from the set $\left\{w_{1}, u_{1}, u_{2}, \ldots, u_{(n-b-1)}, v_{1}\right\}$. Hence $|S| \leq a$. Now, since the set $\left\{w_{1}, v_{1}, v_{2}, \ldots, v_{(a-1)}\right\}$ is an independent position set, we have that $i p(G)=a$.

Theorem 4.3. For every pair of positive integers $a, b$ with $2 \leq a \leq b$, there exists a connected graph $G$ with $i p(G)=a$ and $\alpha(G)=b$.

Proof. We consider the following two cases.

Case 1. $a=b$. Then the the complete bipartite graph $K_{a, a}$ has the required properties.

Case 2. $2<a<b$.

Let $G$ be the graph obtained from the cycle $C_{2(b-a+2)}: v_{1}, v_{2}, \ldots, v_{2(b-a+2)}$ by adding $a-2$ new vertices $u_{1}, u_{2}, \ldots, u_{(a-2)}$ and joining each $u_{i}$ to the vertex $v_{1}$. The graph $G$ thus obtained is shown in the Figure 8. 


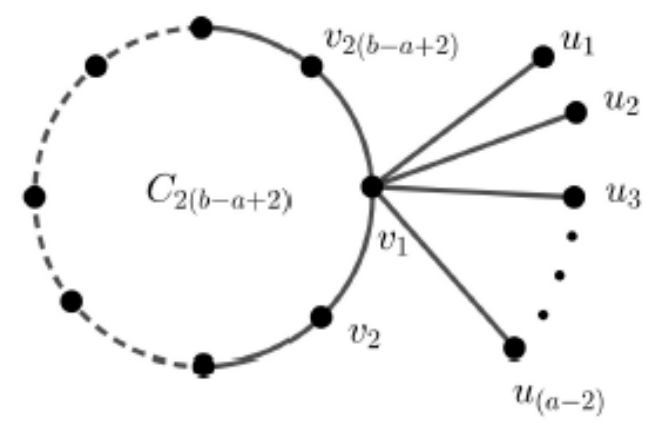

Figure 8

Let $I$ be a maximum independent set in $G$. Then $I$ contains at most $b-a+2$, vertices form the cycle $C_{2(b-a+2)}$. If $v_{1} \in I$, then $|I|=b-a+2 \leq b$. So assume that $v_{1} \notin I$. Then $|I| \leq b$. On the other hand, since the set $\left\{v_{2}, v_{4}, \ldots, v_{2(b-a+2)}, u_{1}, u_{2}, \ldots, u_{(a-2)}\right\}$ is an independent set of size $b$, we have that $|I|=\alpha(G)=b-a+2+a-2=b$.

Next, we prove that $i p(G)=a$. Note that any independent position set $S$ contains at most two vertices from the cycle $C_{2(b-a+2)}$. This shows that $i p(G) \leq a$. Now, since the set $\left\{v_{2(b-a+2)}, v_{2}, u_{1}, u_{2}, \ldots, u_{(a-2)}\right\}$ is an independent position set of size $a$, we have that $\operatorname{ip}(G)=|S|=2+a-2=$ $a$.

\section{References}

[1] P. Manuel and S. Klavžar, "A general position problem in graph theory", Bulletin of the Australian Mathematical Society, vol. 98, no. 2, pp. 177-187, 2018, doi: 10.1017/ S0004972718000473

[2] H. E. Dudeney, Amusements in mathematics. New York, NY: Dover, 1958.

[3] V. Froese, I. Kanj, A. Nichterlein, and R. Niedermeier, "Finding points in general position", International journal of computational geometry \& applications, vol. 27, no. 4, pp. 277-296, 2017, doi: 10.1142/ S021819591750008X 
[4] M. S. Payne and D. R. Wood, "On the general position subset selection problem", SIAM journal on discrete mathematics, vol. 27, no. 4, pp. 1727-1733, 2013, doi: 10.1137/120897493

[5] S. Ullas Chandran and G. J. Parthasarathy, "The geodesic irredundant sets in graphs", International journal of mathematical combinatorics, vol. 4, 2016. [On line]. Available: https:/ / bit.ly/ 3aWmKN3

[6] B. S. Anand, S. Ullas Chandran, M. Changat, S. Klavžar, and E. J. Thomas, "Characterization of general position sets and its applications to cographs and bipartite graphs", Applied mathematics and computation, vol. 359, pp. 84-89, 2019, doi: 10.1016/j.amc.2019.04.064

[7] M. Ghorbani, S. Klavžar, H. R. Maimani, M. Momeni, F. Rahimi Mahid, and G. Rus, "The general position problem on kneser graphs and on some graph operations", Mar. 2019. arXiv: 1903.04286.

[8] B. Patkós, "On the general position problem on kneser graphs", Mar. 2019. arXiv:1903.08056.

[9] P. Neethu, S. Ullas Chandran, M. Changat, and S. Klavžar, "On the general position number of complementary prisms", Jan. 2020. arXiv:2001.02189.

[10] G. M. Thankachy, E. J. Thomas, and S. Ullas Chandran, "On the vertex position number of a graph", unpublished.

[11] E. J. Thomas and S. Ullas Chandran, "Characterization of classes of graphs with large general position number", AKCE international journal of graphs and combinatorics, vol. 17, no. 3, 2020, doi: 10.1016/j.akcej.2019.08.008

[12] R. Hammack, W. Imrich, and S. Klavžar, Handbook of product graphs, 2nd ed. Boca Raton: CRC Press, 2011, doi: 10.1201/ b10959

[13] B. S. Anand, M. Changat, U. Chandran, and P. P. Goswami, "The edge geodetic number of product graphs," in Algorithms and discrete applied mathematics, B. S. Panda, Ed. Cham: Springer, 2018, pp. 143-154, doi: 10.1007/978-3-319-74180-2_12 